\title{
LOCALLY ADAPTIVE VECTOR QUANTIZATION FOR IMAGE COMPRESSION
}

Rodney M. Goodman and Masahiro Sayano

Department of Electrical Engineering

California Institute of Technology

Mail Code 116-81

Pasadena, California 91125 , U. S. A.

\author{
Kar-Ming Cheung and Fabrizio Pollara \\ Jet Propulsion Laboratory \\ 4800 Oak Grove Drive \\ Pasadena, California 91109 , U. S. A.
}

\section{Abstract}

In this paper we study various improvements to a locally adaptive vector quantization (LAVQ) algorithm. The effects of including bit stripping, index compression, and filtering techniques will be discussed. Software implementation and comparisons with non-adaptive vector quantization algorithms will be studied.

\section{LAVQ Algorithm}

The locally adaptive vector quantization (LAVQ) algorithm provides a simple yet effective lossy compression strategy. The encoder has a codebook containing codewords (vectors) where the index of the codeword corresponds to its position in the codebook. A block is taken from the image and compared to the stored codewords; if there exists a codeword sufficiently close to the image block (within the allowed error) the index itself is sent, and that codeword is moved to the top of the codebook. If no such codeword exists, a special index is sent, followed by the actual block itself. This becomes a new codeword, and is placed at the front of the codebook. If the number of codewords exceeds the maximum allowed, the last codeword is lost.

On the decoder side, the decoder expects an index. If this index is the special one, the receiver expects a block to be received immediately following; this block is placed at the top of the code book and all other codewords are pushed down. If the codebook were already full, the last codeword is discarded. This new block is also placed into the image being built by the decoder. If the index is the special one, then the codeword corresponding to the index is put into the image being built, and that codeword is moved to the top of the codebook. Thus, if the encoder and decoder start with the same codebook, they will have the same codebook at each step, and the image will be successfully sent [1][2][3].

The LAVQ strategy maintains the most recently used vector in the codebook in order of last usage; this allows the algorithm to efficiently code any image without codebook training: The LAVQ algorithm needs only one raster-scanned pass of the image to code it entirely. It has time complexity $O(\mathrm{~nm})$ and space complexity $O(m)$, where $n$ is the number of pixels in the image and $m$ is the number of codewords in the codebook. By comparison, standard VQ with hierarchical clustering can have time complexity $O\left(n^{2}\right.$ and space complexity of $O(n)$. However, LAVQ has two major disadvantages, higher rate and staircase effect. The rate for LAVQ is higher than standard VQ for the same codebook size and distortion obtained when using square blocks. Block boundaries are also clearly visible. Both limitations can be reduced dramatically by taking blocks to be tall and narrow $(1 \times \mathrm{N}$ pixels) and by using the methods outlined in the following section.

\section{Improvements to the Algorithm}

\section{Bit Stripping of New Blocks}

The majority of the encoded image is composed of raw blocks being sent when tolerance is exceeded. To reduce the number of bits sent, new blocks are sent stripped of their least significant bits; at the decoder, these stripped bits are replaced by random bits. Since the least significant bits are essentially random for most images, the effect of bit stripping on distortion is not noticeable; also, rate is improved considerably: For one bit stripped off of 8-bit pixels, the decrease can be up to $12.5 \%$ for two bits stripped off, $25 \%$. Bit stripping can help reduce the staircase effect: Since the staircase effect is linked to a slight discontinuity in neighboring pixels at block boundaries, insertion of random bits helps randomize this difference at the block boundaries, making them less pronounced.

\section{Smoothing Filters}

To filter out block boundaries, a bandstop filter with stop bands corresponding to the spacial block frequency and its harmonics can be used [4]. The filter must have smooth transitions to avoid "ringing" at edges in the image, yet must have sufficient selectivity in stopband attenuation to avoid attenuating too many frequencies. Such a filter is effective in reducing the staircase effect, but at the cost of increased fuzziness, especially in high detail regions.

A solution is obtained by replacing those blocks in the filtered image with those which are known to be exactly the same as the original image. Since the encoder sends either indices or a special index followed by the raw unencoded block, the decoder has knowledge of the exact contents of at least a portion of the image. These portions tend to be those in areas of high detail; by replacing the filtered blocks with these "raw" blocks reduces the distortion while maintaining high detail. This replacement strategy cannot be applied to standard VQ algorithms.

\section{One-Pass Index Compression}

Because of the move-to-front codebook rearrangement strategy, the smallest indices are used most often. Therefore, they can be coded using a lossless compression code to obtain better performance. In Elias coding, the most probable elements are those assumed to be those with the smallest indices; coding is done without prior knowledge of codeword statistics. The effects of coding are most significant when allowed error is large-and correspondingly, rate is low and distortion is high.

To maintain one-pass coding, any compression code used on the indices must also be one-pass coding. This means that if Huffman codes are to be used, then the dynamic variant must be implemented. Unfortunately, this code is also computationally intensive. Experimentally, the relation between index and probability of use has been found to be $P(i)=a i^{b} ; i$ is the index; $a$ and $b$ are coefficients determined by multivariable polynomials dependent on codebook size, allowed distortion, and block size. This result allows a static Huffman code to be generated before coding of an image; as a consequence, the algorithm remains very fast. However, since the probabilities are taken as an average over a number of images, it is not optimal, and in some cases, the coding can lead to a drop in performance.

\section{Conclusion}

We have presented a fast and effective vector quantization algorithm which codes images with performance comparable to standard vector quantization algorithms. The rate of LAVQ can be improved and disadvantageous distortion characteristics can be removed by the improvements mentioned, namely bit stripping, filtering, and index compression, without degradation in image quality.

\section{References}

[1] K. M. Cheung and V. K. Wei, "A Locally Adaptive Source Coding Scheme," Proceedings of the Bilkent Conference on New Trends in Communications, Control, and Signal Processing, Ankara, Turkey, July $2-5,1990$.

[2] K. M. Cheung and V. K. Wei, "A Locally Adaptive Source Coding Scheme," submitted IEEE Transactions on Communications, 1990

(3) J. L. Bentley, D. D. Sleator, R. E. Tarjan, and V. K. Wei, "A Locally Adaptive Data Compression Scheme," Communications of the ACM, 29, pp. 320-330, 1986

[4] A. Makur, Low Rate Image Coding using Vector Quantization, Ph.D. thesis, California Institute of Technology, 1990 